\title{
Balancing evidence and opinion in stroke care: the 2008 best practice recommendations
}

\author{
Mark Bayley MD, Patrice Lindsay BScN PhD, Chelsea Hellings BScH, \\ Elizabeth Woodbury BCom MHA, Stephen Phillips MBBS, on behalf of the Canadian Stroke \\ Strategy (a joint initiative of the Canadian Stroke Network and the Heart and Stroke Foundation \\ of Canada)
}

$\mathrm{R}$ esearch in stroke care is generating new information at a rate that challenges our ability to effect healthsystem change in a timely manner. For example, 10 years after publication of a meta-analysis showing that delivery of care in a specialized stroke unit reduced the likelihood of death and disability by up to $30 \%,{ }^{1}$ fewer than one-third of patients with acute stroke who were admitted to Canadian hospitals received this type of specialized care. ${ }^{2}$ The Canadian Stroke Strategy has prepared an update to its 2006 Canadian Best Practice Recommendations for Stroke Care that we hope will help to close this gap in knowledge transfer. In this commentary, we highlight new evidence in stroke care that was incorporated into the recommendations during the update process, discuss areas where it was challenging to find a balance between research evidence and expert opinion, and review the implementation issues identified by a national consensus panel.

In the supplement appearing with this issue of CMAJ (see www.cmaj.ca/content/vol179/issue12/\#supplement), we present best practice recommendations for stroke care in 27 topic areas, 23 updated from the 2006 version of these guidelines and 4 covering new topic areas. ${ }^{3-5}$ Over the past 18 months, expert task groups revised the recommendations released in 2006 and developed the 4 new recommendations. A national consensus panel met in April 2008 to confirm the updated recommendations. Two of the recommendations from the 2006 version of the stroke best practices - those for antiplatelet therapy and acute thrombolysis - required further revision in response to important new information that became available after the April 2008 consensus panel meeting. In both cases, the relevant task groups, consensus panel and other key stakeholders were consulted during the revision process.

The best practice recommendation on antiplatelet therapy for secondary prevention of stroke was modified on the basis of the results of the Prevention Regimen For Effectively avoiding Second Stroke (PRoFESS) trial. ${ }^{6}$ That study did not find a significant difference in stroke recurrence rates between patients treated with the combination of acetylsalicylic acid (ASA) and extended-release dipyridamole and those treated with clopidogrel $(9.0 \%$ v. $8.8 \%$; hazard ratio [HR] $1.01,95 \%$ confidence interval $[\mathrm{CI}] 0.92-1.11)$. This result reinforced the reults of studies comparing ASA with the combination of ASA and extended-release dipyridamole ${ }^{7}$ or ASA with clopidogrel, ${ }^{8}$ which had shown only marginal superiority of these antiplatelet regimens over ASA alone. Therefore, the 2008 best practice recommendation for antiplatelet therapy (recommendation 2.5) emphasizes that patients with ischemic stroke or transient ischemic attack should receive antiplatelet

\section{Key points}

- The 2008 update of the Canadian Best Practice Recommendations for Stroke Care includes 4 new topics: prehospital care, evaluation and treatment of transient ischemic attack and minor stroke, reduction of complications during hospitalization and management of vascular cognitive impairment in stroke.

- The time window for delivery of thrombolytic therapy for acute ischemic stoke has been extended, from 3 to 4.5 hours.

- The recommendations incorporate the themes of discharge planning and stroke in children where appropriate.

therapy unless there is an indication for anticoagulation, and that either ASA, ASA combined with extended-release dipyridamole, or clopidogrel may be used, depending on the clinical circumstances. ${ }^{7,9-11}$ Critics may be concerned that this does not provide sufficient guidance, but the recommendation is intended to convey the importance of sustained long-term antiplatelet therapy while allowing the prescribing physician to tailor treatment to the individual patient.

We also revised the recommendation on thrombolytic therapy (recommendation 3.5) on the basis of data from part 3 of the European Collaborative Acute Stroke Study (ECASS III) ${ }^{12}$ and an updated Cochrane review. ${ }^{13}$ The time window for beneficial treatment of ischemic stroke with intravenous administration of tissue plasminogen activator has been extended from 3 hours to 4.5 hours after onset of stroke symptoms. It is important to emphasize that for a person with stroke, "time is brain," and earlier treatment is associated with better outcomes. Therefore, even if a patient presents later than the 3hour window, he or she should still be considered for acute thrombolysis. However, once a patient does present to the emergency department, he or she should be diagnosed and treated without delay.

We added 4 new topics in this update of the Canadian stroke guidelines. The first of these (recommendation 3.1) relates to the potential of thrombolytic therapy, which has made stroke a time-sensitive emergency. Acute stroke teams and emergency departments must continue to give priority to all suspected stroke patients to ensure rapid diagnosis and treat-

From the Division of Physiatry (Bayley), Toronto Rehabilitation Institute, Toronto, Ont.; the Department of Medicine (Bayley) and the Department of Health Policy, Management and Evaluation (Lindsay, Hellings), University of Toronto, Toronto, Ont.; Canadian Stroke Network (Lindsay), Etobicoke, Ont.; Canadian Stroke Strategy (Woodbury), Ottawa, Ont.; and the Department of Medicine (Neurology) (Phillips), Queen Elizabeth II Health Sciences Centre and Dalhousie University, Halifax, NS 
Box 1: Consensus panel's priorities for implementation of the Canadian best practice recommendations for stroke care

- Management of transient ischemic attack and minor stroke*

- Outpatient and community rehabilitation*

- Development of stroke units*

- Management of stroke by emergency medical services

- Initial assessments for rehabilitation

- Blood pressure management*

- Provision of inpatient rehabilitation*

- Management of post-stroke depression*

- Carotid artery interventions*

- Anticoagulation in stroke patients with atrial fibrillation*

*Supported by the strongest levels of evidence.

ment. A coordinated emergency response system is required to facilitate access to thrombolytic therapy. Transport of stroke patients to hospitals equipped to manage them is expedited when well-trained emergency personnel follow agreedupon protocols. ${ }^{14}$ We consider this a key component of organized stroke care. We have therefore recommended, on the basis of observational evidence alone, that "all suspected stroke patients with symptom onset within the previous 4.5 hours must be treated as time-sensitive emergency cases and should be transported without delay to the closest institution that provides emergency stroke care."

The second new recommendation (recommendation 3.2) concerns the management of patients who present to primary care or the emergency department with suspected transient ischemic attack or minor stroke. The risk of a disabling stroke, approximately 5\% in the first week after transient ischemic attack, can be substantially reduced by timely diagnosis and intervention..$^{15} \mathrm{~A}$ prospective population-based, sequential comparison study (the Early use of eXisting PREventive Strategies for Stroke or EXPRESS study) showed that the early use of existing preventive strategies in the setting of a specialized clinic reduced by $80 \%$ the risk of disabling stroke 3 months after a transient ischemic attack or minor stroke. ${ }^{16}$ Although this was not a randomized controlled trial, the methodologic rigour of the study was considered sufficient to justify the recommendation that patients with transient ischemic attack should be referred immediately to a physician specializing in stroke management in a stroke prevention clinic or, if these options are unavailable, to an emergency department with access to neurovascular imaging facilities.

During our consultation process over the past couple years, several stakeholders requested further guidance on specific aspects of inpatient stroke care. Although the impact of stroke unit care on death and disability appears to be mediated through the prevention of post-stroke complications and the early initiation of coordinated interdisciplinary rehabilitation, ${ }^{17}$ many components of early stroke care have not been well studied. Early mobilization, the prevention of venous thromboembolism, management of oral care, provision of nutritional support, assessment of dysphagia, and management of fever and of bladder and bowel function emerged from our consultation process as new additions, together forming the third new best practice recommendation (recommendation 4.2). Again, although the strength of the research evidence varied across these topics, they were included because of their potential impact on recovery and length of stay in hospital.

Emerging research suggests that for every clinically evident stroke, there are 6 to 9 "covert" strokes that may manifest as cognitive impairment. ${ }^{18}$ Vascular cognitive impairment affects up to $60 \%$ of stroke survivors and is associated with decreased function in activities of daily living. ${ }^{19}$ The fourth new recommendation (recommendation 6.3) therefore addresses the assessment and management of vascular cognitive impairment. The challenge for the consensus panel was to balance the magnitude of the problem of vascular cognitive impairment against the relative paucity of evidence to guide management. The resulting recommendation aims to bring attention to vascular cognitive impairment, the importance of aggressive management of stroke risk factors and the need for further research in this area.

\section{Implementation}

The work of the 2008 consensus panel included identification of the barriers, enablers and priorities for implementation of the Canadian Best Practice Recommendations for Stroke Care. The 2 most significant barriers identified by the panel were (1) competing priorities within health care systems, regions and institutions and (2) limited human, financial and equipment resources. The consensus panel was asked to prioritize the recommendations for implementation. Interestingly, when asked the question: "Which 10 recommendations, if implemented immediately, would have the greatest impact on stroke care in Canada?" the panel selected 8 recommendations with the highest levels of evidence and 2 key areas that do not yet have strong research evidence but are considered significant stroke system drivers (Box 1). Recovery after stroke features prominently in these 10 priority areas because of the recognition that in-hospital and community rehabilitation improves outcomes.

\section{Next steps}

The Canadian Best Practice Recommendations for Stroke Care aim to provide clear direction for clinical care and systems change. The limited number of high-quality studies in some areas led to tensions between research evidence and expert opinion during the guideline development process. Nonetheless, of the 47 individual components of the recommendation statements, 25 are supported by randomized controlled trials, 15 are based on nonrandomized controlled trials and 7 are based on expert consensus. Thus, our synthesis of the evidence identified a number of key areas where additional research is needed. We believe that the immediate priorities in stroke care should be the widespread implementation of the best practice recommendations outlined in the 2008 update 
and a systematic evaluation of their impact on processes of care, patient outcomes and system functioning. System-wide measures of processes of care and clinically important outcomes will provide clear direction for ongoing efforts to improve the quality and equity of stroke care across Canada.

This article has been peer reviewed.

Competing interests: None declared.

Contributors: All of the authors contributed to the content of the article, revised it critically and approved the final version submitted for publication.

\section{REFERENCES}

1. Langhorne P, Williams BO, Gilchrist W, et al. Do stroke units save lives? Lancet 1993;342:395-8

2. Kapral MK, Laupacis A, Phillips SJ, et al.; Investigators of the Registry of the Canadian Stroke Network. Stroke care delivery in institutions participating in the Registry of the Canadian Stroke Network. Stroke 2004;35:1756-62.

3. Canadian Stroke Network; Heart and Stroke Foundation of Canada. Canadian best practice recommendations for stroke care 2006. Ottawa (ON): Canadian Stroke Strategy; 2006. Available: www.canadianstrokestrategy.ca/eng/resourcestools/documents /StrokeStrategyManual.pdf (accessed 2008 Oct 28).

4. Lindsay P, Bayley M, McDonald A, et al. Toward a more effective approach to stroke: Canadian Best Practice Recommendations for Stroke Care. CMAJ 2008; 178:1418-25.

5. Lindsay $\mathrm{P}$, Bayley $\mathrm{M}$, Hellings $\mathrm{C}$, et al. Canadian best practice recommendations for stroke care (updated 2008). CMAJ Available: www.cmaj.ca/content/vol179 /issue12/\#supplement.

6. Sacco R, Diener HC, Yusuf S, et al.; PRoFESS Study Group. Aspirin and extended-release dipyridamole versus clopidogrel for recurrent stroke. $N$ Engl J Med 2008;359:1238-51

7. Verro P, Gorelick PB, Nguyen D. Aspirin plus dipyridamole versus aspirin for prevention of vascular events after stroke or TIA: a meta-analysis. Stroke 2008;
39:1358-63

8. Hankey GJ, Sudlow CL, Dunbabin DW. Thienopyridine derivatives (ticlopidine, clopidogrel) versus aspirin for preventing stroke and other serious vascular events in high vascular risk patients. Cochrane Database of Syst Rev 2000;(2):CD001246.

9. CAPRIE Steering Committee. A randomised, blinded, trial of clopidogrel versus aspirin in patients at risk of ischaemic events (CAPRIE). Lancet 1996;348:1329-39.

10. Halkes PH, van Gijn A, Kappelle LJ, et al.; ESPRIT Study Group. Aspirin plus dipyridamole versus aspirin alone after cerebral ischaemia of arterial origin (ESPRIT): randomised controlled trial. [published erratum in Lancet 2007;369:274]. Lancet 2006:367:1665-73.

11. De Schryver EL, Algra A, van Gijn J. Dipyridamole for preventing stroke and other vascular events in patients with vascular disease. An update. In: The Cochrane Library, Issue 3. Oxford (UK): Update Software. MetaView; 2007.

12. Hacke W, Kaste M, Bluhmki E, et al.; ECASS Investigators. Thrombolysis with alteplase 3 to 4.5 hours after acute ischemic stroke. N Engl J Med 2008;359:1317-29.

13. Mielke O, Wardlaw J, Liu M. Thrombolysis (different doses, routes of administration and agents) for acute ischaemic stroke. Cochrane Database Syst Rev 2004;(2):CD000514

14. Riopelle RJ, Howse DC, Bolton C, et al. Regional access to acute ischemic stroke intervention. Stroke 2001;32:652-5.

15. Giles MF, Rothwell PM. Risk of stroke early after transient ischaemic attack: systematic review and meta-analysis. Lancet Neurol 2007;6:1063-72.

16. Hackam DG, Spence JD. Combining multiple approaches for the secondary prevention of vascular events after stroke: a quantitative modeling study. Stroke 2007;38:1881-5.

17. Langhorne P, Pollock A; Stroke Unit Trialists' Collaboration. What are the components of effective stroke unit care? Age Ageing 2002;31:365-71.

18. Teasell RW, Foley NC, Salter K, et al. EBRSR: evidence-based review of stroke re habilitation. 10th ed. London (ON): EBRSR; 2007. Available: www.EBRSR.com/ (accessed 2008 Oct 28)

19. Black SE. Therapeutic issues in vascular dementia: studies, designs and approaches. Can J Neurol Sci 2007;34 Suppl 1:S125-30.

Correspondence to: Dr. Mark Bayley, Toronto Rehabilitation Institute, Division of Physiatry, 550 University Ave., Rm. 1023, Toronto ON M5G 2A2; fax 416 597-3422 x3943;

Bayley.Mark@TorontoRehab.on.ca

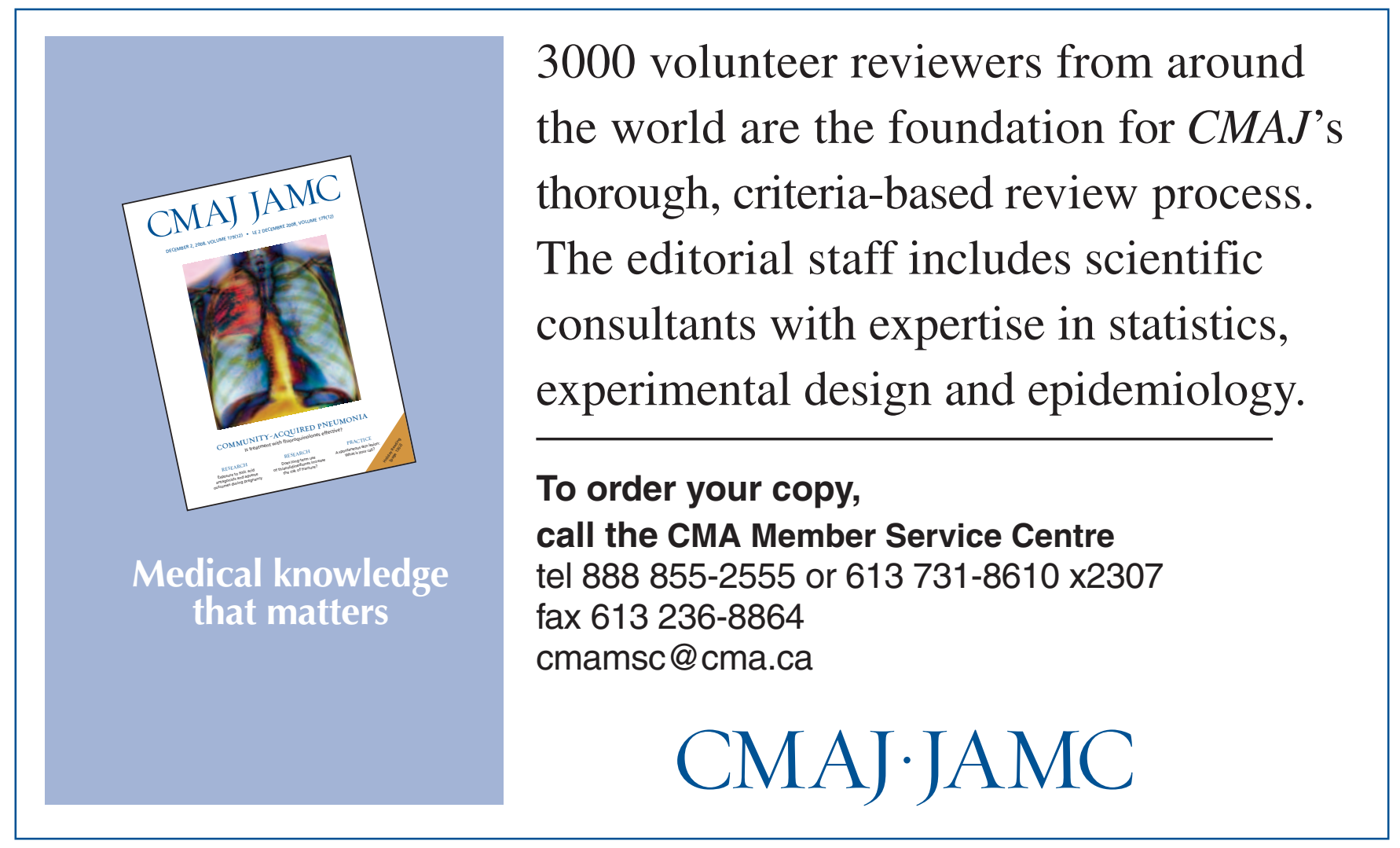

